\title{
LA CIENCIA Y LA PAZ
}

\section{THE SCIENCE AND THE PEACE}

\author{
DE LA OSSA, V. JAIME ${ }^{\star *}$ Dr. Sci. \\ ${ }^{1}$ Universidad de Sucre, Facultad de Ciencias Agropecuarias, Grupo de \\ Investigación en Biodiversidad Tropical. Editor Revista Colombiana de Ciencia \\ Animal - RECIA, Colombia.
}

*Correspondencia: jaimedelaossa@yahoo.com

La Organización de las Naciones Unidas para la Educación, la Ciencia y la Cultura (UNESCO), en el año 2001, en su Conferencia General en la Resolución 31C/20 decidió proclamar el día 10 de noviembre como el día mundial de la ciencia para la paz y el desarrollo. Se trata de un evento anual celebrado en todo el mundo para recordar el compromiso asumido en la Conferencia Mundial sobre la Ciencia, celebrado en Budapest en 1999, con el auspicio de la UNESCO y el Consejo Internacional de Uniones Científicas (CIUC).

El propósito de este conmemorativo día busca renovar el compromiso, tanto nacional como internacional, que debe existir entre la ciencia para la paz y el desarrollo, hace hincapié en la utilización responsable de la ciencia en beneficio de las sociedades y en particular, para la erradicación de la pobreza y en pro de la seguridad humana. Este día Mundial también tiene por objeto lograr una mayor conciencia en el público, resaltando la importancia de la ciencia y su papel para disminuir la brecha existente entre la ciencia y la sociedad. Tal como acertadamente lo señala la directora general de la UNESCO, IRINA BOKOVA, al convocar a la comunidad internacional a "aprovechar el potencial de las ciencias en beneficio de la paz duradera y el desarrollo sostenible".

No se debe olvidar, que en términos generales y referentes a Ciencia, el intercambio de datos, conocimientos e información es esencial para la gestión sostenible de los recursos naturales. Pero también es imprescindible que exista por parte del Estado una posición crítica y reivindicatoria para evitar la posición monopólica de las grandes editoriales internacionales que concentran la publicación de investigaciones científicas, que cobran por ellas y solo liberan estas cuando su utilidad no riñe con sus intereses económicos y de dominación. El objetivo de la producción científica financiada por la sociedad debe ser la accesibilidad libre. El planteamiento debe ir más allá de lo dicho, se deben fomentar y financiar las bases de datos nacionales, al igual que las revistas y 
medios de divulgación que sean propios de la Ciencia, para que sea una realidad total la disposición y uso de los hallazgos científicos nacionales, que sin duda contribuyen a mantener un Estado de paz y desarrollo.

Sin perder la rigurosidad que caracteriza a la Ciencia, se debe obligar a publicar nacionalmente todos aquellos escritos que se deriven de investigaciones financiadas directa 0 indirectamente con fondos del Estado, bien sean fondos relacionados con proyectos, así como por los salarios o pagos que reciban los investigadores o por los reconocimientos económicos que las instituciones otorgan en tal sentido. Las publicaciones, si es el caso, pueden hacerse nacionalmente en cualquier idioma, si es que la excusa es la visibilidad, pueden hacerse virtualmente para que no tengan barreras geográficas. No se debe permitir que las revistas nacionales se extingan.

De manera particular y exaltando la ética posición del Doctor FELIPE GARCíA VALLEJO, se insertan, de su análisis sobre la situación actual de la investigación en Colombia, en relación con la convocatoria pública 640: "Convocatoria nacional para el reconocimiento y medición de grupos de investigación, desarrollo tecnológico y/o innovación y para el reconocimiento de investigadores del sistema nacional de ciencia, tecnología e innovación. 2013", los siguientes análisis, que demuestran que en Colombia, contrariamente a los que propone la UNESCO en materia de ciencia, paz y desarrollo, se inicia un recorrido en contravía, que generará caos y posiblemente atrasará al país:

1. "Dicha Convocatoria solo permitirá poner a prueba un modelo de medición de grupos el cual de entrada tiene falencias conceptuales, es simplista y promueve la inequidad. Además, desconoció las manifestaciones de rechazo y objeciones de fondo que los investigadores y académicos del país hicieron al modelo de medición propuesto por COLCIENCIAS".

2. "Sin la introducción de los incentivos y correcciones pertinentes y contra toda lógica y siguiendo las directrices erróneas que buscan que Colombia sea en un mediano plazo miembro de la OECD (Organisation for Economic Cooperation and Development), se inicia el lento pero progresivo exterminio de los grupos de investigación en nuestro país y de las revistas nacionales. De hecho el 25 de octubre del presente año se lanzó oficialmente la hoja de ruta para el proceso de adhesión de Colombia a la Organización, el cual debe darse sobre las condiciones de la OECD en un lapso aproximado de tres años". 
3. "De la lectura hecha al documento "Estudios de la OECD de las políticas de innovación: Colombia. Evaluación general y recomendaciones", queda claro que las recomendaciones hechas por esta organización se refieren exclusivamente a la implementación de un modelo de Ciencia modo 2, con una fuerte tendencia a la innovación empresarial y a la organización del sistema con base en una política mercantilista que busca la innovación en las empresas dejando de lado otras instituciones que como las Universidades, los Institutos y Centros de investigación puedan hacerla desde una investigación básica rigurosa y cumpliendo con los ciclos de evolución hacia la innovación tecnológica".

4. "Queda claro que el objetivo central, de acuerdo con COLCIENCIAS, es probar un instrumento que "mida" la capacidad del sistema para generar conocimiento principalmente en la innovación empresarial y el desarrollo tecnológico ligado a las empresas (de nuevo Ciencia modo 2), ignorando logros históricos de la academia en el reconocimiento a una investigación básica de excelencia como factor de desarrollo con equidad social y al esfuerzo editorial de muchas universidades por mantener una actividad de difusión de los productos de generación de conocimiento contextualizados en el entorno nacional y con seriedad académica. En este escenario, es claro que la aplicación del modelo de medición de grupos 2013 que se hará en la convocatoria 640, producirá un efecto de extinción masiva de la ya débil arquitectura de los grupos de investigación en nuestro país".

En otro de los apartes del documento presentado por el Dr. FELIPE GARCíA VALLEJO, en relación con el instrumento de medición que usará COLCIENCIAS y teniendo como base el estudio llevado a cabo por JOHAN BOLLEN y colaboradores del "Digital Library Research and Prototyping Team, Research Library", de "Los Alamos National Laboratory", en Los Alamos, NM, de Estados Unidos cuyo título en Inglés es "A Principal Component Analysis of 39 Scientific Impact Measures" publicado en la revista PLoS ONE 4(6): e6022 del 2009, señala que los resultados indicaron que la noción de impacto científico es un construido multidimensional que no se puede medir adecuadamente con la aplicación de un solo indicador, sino con una medición de conjuntos de indicadores bibliométricos disponibles. Las conclusiones de este estudio, refirman el postulado que el modelo de medición 2013 que aplicará COLCIENCIAS, es lineal y simplista, aunque use los ya tradicionales cuestionados indicadores de medición. ¿Será que sobre la base de este y otros análisis, los cuales cuestionan la métrica unidimensional, se podrán obtener resultados que midan el impacto real de las publicaciones provenientes de los grupos de investigación solo con base en los indicadores propuestos por Colciencias? Es preocupante analizar cómo un instrumento de medición pueda condenar a la desaparición de muchos grupos de investigación, 
principalmente aquellos de las Ciencias Sociales y Humanas, las Artes, la Filosofía y aun varios que trabajan en las áreas de las Ingenierías y las Ciencias Físicas, Exactas y Naturales.

Quedan por analizar diversos escenarios, uno de ellos de gran actualidad y que sin duda cambiara la dinámica nacional en todos los aspectos, es la firma de la Paz, proceso que se adelanta y que se aspira llegará a concretarse. Entonces: ¿Cómo será la Ciencia en un país que iniciará una reconstrucción social en lo que se ha llamado el posconflicto? ¿Cómo abordarán de forma responsable el Estado y las Universidades la investigación científica, si el modelo que se implantará antes del acuerdo de paz es inequitativo y discriminador? ¿Cómo acordarán las partes, gobierno y FARC, un modelo de ciencia para la paz y el desarrollo?

Solo esperamos que haya corrección y que se preserve una amplia y diversa investigación científica nacional, que se valore el esfuerzo que hoy hacen muchos investigadores, académicos y científicos en todo el territorio colombiano, cuya tenacidad está mostrando avances a instancias de todas las dificultades que se tienen para hacer ciencia libre, para construir ciencia que ofrezca paz y desarrollo. No se puede permitir que se destruya el ingente esfuerzo que ha costado crear y mantener múltiples grupos de investigación y que ha generado, igualmente, apreciable número de valiosas revistas científicas que divulgan resultados importantes, que son apreciable producto de la capacidad nacional; antes que desaparecer los grupos y las revistas, deberían incentivarse y financiarse, medida trascendental para evitar su extinción y hacerlos cada vez más representativos, como debe ser... COLCIENCIAS TIENE LA PALABRA. 